\title{
The p.G534E variant of HABP2 is not associated with sporadic papillary thyroid carcinoma in a Polish population
}

\author{
Artur Kowalik ${ }^{1,2}$, Danuta Gąsior-Perczak³, Martyna Gromek ${ }^{1}$, Monika Siołek ${ }^{4}$,

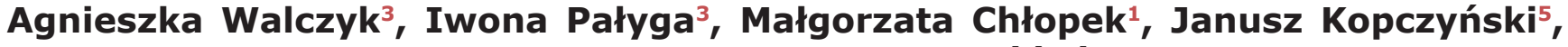 \\ Ryszard Mężyk ${ }^{6}$, Aldona Kowalska ${ }^{3}$ and Stanisław Góźdź7,8 \\ ${ }^{1}$ Department of Molecular Diagnostics, Holycross Cancer Centre, Kielce, Poland \\ ${ }^{2}$ Department of Surgery and Surgical Nursing with The Scientific Research Laboratory, The Faculty of Health Sciences of The \\ Jan Kochanowski University, Kielce, Poland \\ ${ }^{3}$ Endocrinology Clinic, Holycross Cancer Centre, Kielce, Poland \\ ${ }^{4}$ Genetic Clinic, Holycross Cancer Centre, Kielce, Poland \\ ${ }^{5}$ Department of Surgical Pathology, Holycross Cancer Centre, Kielce, Poland \\ ${ }^{6}$ Cancer Epidemiology, Holycross Cancer Centre, Kielce, Poland \\ ${ }^{7}$ Oncology Clinic, Holycross Cancer Centre, Kielce, Poland \\ ${ }^{8}$ The Faculty of Health Sciences, Jan Kochanowski University, Kielce, Poland \\ Correspondence to: Artur Kowalik, email: arturko@onkol.kielce.pl \\ Keywords: p.G534E, sporadic papillary thyroid carcinoma, HABP2, thyroid cancer, non-medullary thyroid cancer \\ Received: October 20, $2016 \quad$ Accepted: March 09, $2017 \quad$ Published: April 06, 2017 \\ Copyright: Kowalik et al. This is an open-access article distributed under the terms of the Creative Commons Attribution License 3.0 \\ (CC BY 3.0), which permits unrestricted use, distribution, and reproduction in any medium, provided the original author and source \\ are credited.
}

\section{ABSTRACT}

Thyroid cancer is one of the most frequently diagnosed cancers of the endocrine system. There are no known genetic risk factors for non-medullary thyroid cancer, other than a small number of hereditary syndromes; however, approximately $5 \%$ of non-medullary thyroid cancer, designated familial non-medullary thyroid cancer, exhibits heritability. The p.G534E (c.1601G>A) variant of HABP2 was recently reported as a risk factor for familial non-medullary thyroid cancer, including papillary thyroid carcinoma. We analyzed the incidence of the c.1601G $>A$ variant of HABP2 in a Polish population consisting of 326 cases of papillary thyroid carcinoma and 400 control individuals by DNA genotyping, performed by Sanger sequencing. The c.1601G > A variant was detected in $3.7 \%$ of sporadic papillary thyroid carcinoma cases and $4.7 \%$ of healthy controls, and we did not detect an association between this variant and sporadic papillary thyroid carcinoma risk $(O R=0.71,95 \% \mathrm{CI}$ : $0.33-1.51$; $p=0.3758$ ). Additionally, no significant associations were identified between clinical and pathological disease features, response to primary treatment, and clinical status at the end of the observation, and HABP2 c.1601G>A genotype. In conclusion, the p.G534E variant of $H A B P 2$ is not associated with sporadic papillary thyroid carcinoma risk in the Polish population.

\section{INTRODUCTION}

Thyroid cancer (TC) is one of the most frequently diagnosed endocrine malignancies worldwide [1] and its incidence is rising in Poland and elsewhere [2]. Other than hereditary syndromes (Cowden Syndrome, Carney Complex, Werner Syndrome), there are no known genetic risk factors for the development of non-medullary thyroid cancer (NMTC); however, approximately $5 \%$ of NMTC appears to exhibit heritability, and is classified as familial non-medullary thyroid cancer (FNMTC) [3]. Among NMTC cases, $85 \%$ are papillary thyroid cancer (PTC) [4]. Recently, Gara et al. described a multigenerational family cosegregating the $\mathrm{c} .1601 \mathrm{G}>\mathrm{A}$ 
Table 1: Details of PTC patients and controls

\begin{tabular}{|c|c|c|c|c|}
\hline & PTC patients & Controls & Test & Significance \\
\hline Number of samples & 326 & 400 & Reference & \\
\hline Number with genotype, $\mathbf{A A}$ & 1 & 0 & NA & NA \\
\hline Number with genotype, GA & 11 & 19 & Logistic regression & $\begin{array}{c}\text { OR }(95 \% \mathrm{CI}) \\
0.71(0.33-1.51) \\
p=0.3758\end{array}$ \\
\hline $\begin{array}{l}\text { Percentage with genotypes } \\
\text { GA }+\mathbf{A A}\end{array}$ & 3.7 & 4.7 & $\begin{array}{l}\text { Fisher's exact test } \\
\text { (vs. GG genotype) }\end{array}$ & $p=0.580902$ \\
\hline Mean age (SD) & $53.5(13.5)$ & $53.6(9.9)$ & $\begin{array}{c}\text { Independent Sample } \\
\text { t-test }\end{array}$ & $p=0.8461$ \\
\hline $\mathbf{F}: \mathbf{M}$ & 296:30 (90.8\%:9.2\%) & $316: 84(70 \%: 30 \%)$ & $\chi^{2}$ & $p<0.0001$ \\
\hline
\end{tabular}

F, female; M, male; SD, standard deviation; $\chi 2$, Chi-squared test, NA, not applicable.

variant of $H A B P 2$ (rs7080536; p.G534E), which sparked hope of a new genetic marker that could be useful for TC patient stratification and clinical management. Their investigations also demonstrated a role for $H A B P 2$ as a tumor suppressor, suggesting that the identified variant is pathogenic [5]. Since then, several papers have reported an inability to confirm the importance of the p.G534E variant of $H A B P 2$ in the development of FNMTC [7-11], with only one report supportive of the association [6]. Although research has been conducted using data from European populations (English and Spanish), there is a lack of information about the prevalence of this variant in PTC patient populations in Eastern Europe [12, 13]. Therefore, we assessed the prevalence of the c. $1601 \mathrm{G}>\mathrm{A}$ variant of $H A B P 2$ in patients with sporadic PTC and a control Polish population, characterized by high homogeneity.

\section{RESULTS}

Using Sanger sequencing, we detected the c. $1601 \mathrm{G}>\mathrm{A}$ variant of $H A B P 2$ in $12 / 326(3.7 \%)$ sporadic PTC patients; 11 (3.4\%) carried the heterozygous (GA) and one $(0.3 \%)$ the homozygous (AA) genotype at this locus. In the control group, the heterozygous GA genotype was detected in 19/400 (4.75\%) patients with no homozygotes detected (Table 1). Statistical analysis indicated no significant difference in the incidence of the variant between the PTC and control populations (Fisher's exact test, $p=0.580902$ ), and we did not detect an association between the c. $1601 \mathrm{G}>\mathrm{A}$ variant of $H A B P 2$ and risk of sporadic PTC by logistic regression analysis $(\mathrm{OR}=0.71,95 \%$ CI: $0.33-1.51, p=0.3758)$.

In addition, we assessed the correlation between clinical and pathological disease features, response to primary treatment, and clinical status at the end of the observation and detected $H A B P 2$ genotypes (GG, GA, AA) in the PTC patient group. The results revealed no statistically significant differences (Table 2). In the group of sporadic PTC patients, only one patient (with a GG genotype at the $H A B P 2$ variant) was classified as FNMTC according to the algorithm applied by our Outpatient Genetic Clinic (FNMTC was defined as the presence of at least three cases of PTC in a family). In addition, the homozygous AA genotype was detected in only one 63-year-old female patient with sporadic PTC.

\section{DISCUSSION}

The basis for the inherited genetic predisposition of FNMTC is largely unknown. Recently, Gara et al. published the results of a study indicating a relationship between the c.1601G $>$ A (rs7080536; G534E) variant in the $H A B P 2$ gene and PTC in a large multigenerational family [5]. All but one [6] subsequent report failed to confirm these results in analyses of dozens of families, including large families from different populations (Spain, USA, Australia, and the Middle East), that did not identify cosegregation of the $\mathrm{c} .1601 \mathrm{G}>\mathrm{A}$ variant of $H A B P 2$ with PTC [11, 13-15].

There is some controversy regarding the definition of FNMTC, which can be designated by as few as two cases of TC in the immediate family. Due to the high incidence of TC, it is possible for two cases of the disease to be a chance occurrence; hence diagnosis of FNMTC on the basis of only two cases may be incorrect $[16,17]$, while extension of the definition to require a minimum of three cases in a family improves specificity [18]. In the one report supportive of the $H A B P 2$ association [6], two of four analyzed families included more than two cases of PTC (first- or second-degree relatives), while in the remaining FNMTC families there were only two cases of PTC, including one in a cousin (second-degree relative). In the present study, only one patient was from a FNMTC family, and we did not detect the c. $1601 \mathrm{G}>\mathrm{A}$ variant of $H A B P 2$ in the proband. 
Table 2: Evaluation of associations between detected $H A B P 2$ genotypes (GG, GA, and AA) and clinical variables

\begin{tabular}{|c|c|c|c|c|c|}
\hline \multirow{2}{*}{ Characteristic } & & \multicolumn{3}{|c|}{ Genotype } & \multirow{2}{*}{$\begin{array}{c}\text { Statistical } \\
\text { significance }\end{array}$} \\
\hline & & GG & $\mathbf{A A}$ & GA & \\
\hline Gender & $\mathrm{F} / \mathrm{M}$ & $286 / 28$ & $1 / 0$ & $10 / 1$ & NS \\
\hline Median tumor diameter (mm) & & 8 & 25 & 13 & NS \\
\hline Age (years) & & 50 & 62 & 37 & NS \\
\hline \multirow[t]{4}{*}{ Tumor stage } & $\mathrm{T} 1$ & 228 & 0 & 7 & NS \\
\hline & $\mathrm{T} 2$ & 19 & 0 & 1 & \\
\hline & T3 & 54 & 1 & 3 & \\
\hline & $\mathrm{T} 4$ & 13 & 0 & 0 & \\
\hline \multirow[t]{3}{*}{ Lymph node involvement } & No & 123 & 1 & 5 & NS \\
\hline & N1 & 28 & 0 & 0 & \\
\hline & $\mathrm{Nx}$ & 163 & 0 & 6 & \\
\hline \multirow[t]{2}{*}{ Metastasis } & M0 & 313 & 1 & 11 & NS \\
\hline & M1 & 1 & 0 & 0 & \\
\hline \multirow[t]{2}{*}{ Extra-thyroidal invasion } & Yes & 63 & 0 & 2 & NS \\
\hline & No & 251 & 1 & 9 & \\
\hline \multirow[t]{2}{*}{ Multifocality } & Yes & 261 & 1 & 8 & NS \\
\hline & No & 53 & 0 & 3 & \\
\hline \multirow[t]{3}{*}{ PTC type } & Classical & 276 & 1 & 7 & NS \\
\hline & Follicular & 35 & 0 & 4 & \\
\hline & Oxyphilic & 3 & 0 & 0 & \\
\hline \multirow[t]{4}{*}{ Response to therapy } & Excellent & 280 & 1 & 10 & NS \\
\hline & Indeterminate & 14 & 0 & 1 & \\
\hline & Biochemically incomplete & 3 & 0 & 0 & \\
\hline & Structurally incomplete & 17 & 0 & 0 & \\
\hline Follow-up (years) & Median (Min-Max) & $7(1-48)$ & $6(6-6)$ & $5(3-15)$ & NS \\
\hline \multirow[t]{6}{*}{ Status of final follow-up } & No evidence of disease & 295 & 1 & 10 & NS \\
\hline & Biochemically persistent disease & 17 & 0 & 1 & \\
\hline & Persistent structural disease & 1 & 0 & 0 & \\
\hline & Recurrence & 1 & 0 & 0 & \\
\hline & Death & 0 & 0 & 0 & \\
\hline & Total & 314 & 1 & 11 & \\
\hline
\end{tabular}

Associations between genotypes and clinical variables were assessed using $\chi^{2}$ and Kruskal-Wallis tests. NS, not statistically significant; F, female; M, male. 
Gara et al. [5] detected the p.G534E variant at a frequency of $4.7 \%$ (19 of 423) in data from multi-ethnic sporadic PTC patients in the Cancer Genome Atlas (TCGA), compared with $0.7 \%$ of those with unknown disease status. Immediately following their report, several groups were unable to repeat their findings; the reported frequencies of the p.G534E polymorphism in different NMTC populations (from China, the Middle East, Columbia, the UK, Australia, and the USA) vary from $0 \%$ to $7.8 \%$. Similarly, the frequencies of the polymorphism in control groups from the same populations range from $0 \%$ to $9.3 \%$. In all of these populations, no significant differences were identified in the frequency of the p.G534E variant of $H A B P 2$ between NMTC and control groups $[9,11-15,19]$. In the present study of patients with sporadic PTC, the $\mathrm{c} .1601 \mathrm{G}>\mathrm{A}$ variant of $H A B P 2$ was detected in $3.7 \%$ of cases, while it was present in $4.75 \%$ of controls. Hence, we did not detect an association between the p.G534E variant of $H A B P 2$ and sporadic PTC risk; nor was there any association of this variant with any clinical variables included in the study (Tables 1 and 2). Similarly, other published studies failed to detect associations of the variant with NMTC risk or other clinical variables $[12,19]$. The reported absence of the p.G534E variant of $H A B P 2$ in a Middle Eastern pediatric and juvenile NMTC population (5-26 years old) provides additional evidence that the variant is not associated with this disease $[13,15]$.

According to 1000 genomes project data (http:// www.1000genomes.org/), the c. $1601 \mathrm{G}>\mathrm{A}$ variant of $H A B P 2$ is present in $5.2 \%$ of Europeans. In the present study of a Polish population, the variant frequency was $4.75 \%$, placing it in the middle of the range of values observed in other European populations (Finnish, 7.1\%; British, 9.9\%; Iberian, 2.8\%; and Toscani (Italian), 2.8\%).

In conclusion, based on our results, the p.G534E variant of $H A B P 2$ is not associated with sporadic PTC and is not a risk factor for PTC development in a homogeneous Eastern European Polish population.

\section{MATERIALS AND METHODS}

\section{Patients and controls}

This study included 326 patients with sporadic PTC. Their mean age at diagnosis was 53.5 years (SD, 13.5 years), and one tenth of them were male (Table 1). A group of 400 healthy individuals (mean age, 53.6 years; SD, 9.9 years) was included in the study as a control cohort. There was no significant difference in age between cases and controls $(p=0.8461)$. All cases and controls were recruited at the Holycross Cancer Center in Kielce. All study participants provided written informed consent before participating in the research. All study procedures were and performed according to Declaration of Helsinki.

\section{Materials}

Genomic DNA isolated from whole blood samples, obtained from all study participants, was used for genetic analyses. Material was extracted using a commercial DNA extraction kit (Genomic Micro AX BLOOD Gravity; A \& A Biotechnology, Gdańsk, Poland), according to the manufacturer's instructions. DNA quality and purity were verified using a NanoDrop spectrophotometer (Thermo Fisher Scientific).

\section{Sanger sequencing}

HABP2 mutation analysis was performed by sequencing of PCR products. A target DNA region (181 bp) was amplified using KAPA SYBR Fast DNA Polymerase (KAPA BIOSYSTEMS) and the following oligonucleotide primer pair: forward 5'-TCTCTGGTTCACGAGGATGA-3' and reverse 5'-GGCTTTGATCCAATTCAGGA-3'. The amplification conditions consisted of an initial denaturation at $95^{\circ} \mathrm{C}$ for $3 \mathrm{~min}$, then 35 cycles of denaturation at $95^{\circ} \mathrm{C}$ for $30 \mathrm{~s}$, annealing at $65^{\circ} \mathrm{C}$ for $30 \mathrm{~s}$, and extension at $72^{\circ} \mathrm{C}$ for $20 \mathrm{~s}$, followed by a final extension at $72^{\circ} \mathrm{C}$ for 2 min. PCR was performed using a Veriti Thermal Cycler (AppliedBiosystems). PCR products were separated by microchip electrophoresis, using MultiNA system (Shimadzu Corporation, SHIM-POL, Poland). Amplified material was purified using FastAP and Exonuclease I (Thermo Fisher Scientific), according to the manufacturer's protocol. Sequencing was carried out using the same forward primer that was used for PCR amplification (see above). The primer was diluted at a ratio of $8: 42 \mu \mathrm{l}$ in water. Other sequencing reagents were as follows: BigDye ${ }^{\circledR}$ Terminator v1.0 or v3.0 and 5X Sequencing Buffer (Thermo Fisher Scientific). The sequencing reaction was performed using the Veriti Thermal Cycler (Applied Biosystems) and the following conditions: 25 cycles at $96^{\circ} \mathrm{C}$ for $10 \mathrm{~s}, 50^{\circ} \mathrm{C}$ for $5 \mathrm{~s}$, and $60^{\circ} \mathrm{C}$ for $1 \mathrm{~min} 45 \mathrm{~s}$. The reactions were then purified using a BigDye XTerminator ${ }^{\circledR}$ Purification Kit (SAM Solution and XTerminator Solution; Thermo Fisher Scientific). Next, products were separated and analyzed using an ABI 3130 Genetic Analyzer (Applied Biosystems). The resulting DNA sequences were analyzed using BLAST (www.blast.ncbi.nlm.nih.gov).

\section{Statistical analyses}

The mean age of cases and controls was compared using an Independent Samples t-test. To assess the predictive power of the genotypes for PTC risk (odds ratios, ORs), logistic regression was performed. Differences in the frequencies of genotypes between cases and controls were determined using a two-tailed Fisher's exact test. Associations between genotypes and clinical variables were assessed using $\chi^{2}$ and Kruskal-Wallis tests. Association between sexes and study groups was assessed using $\chi^{2}$. Statistical significance was set at $p<0.05$. 


\section{Abbreviations}

FNMTC: familial non-medullary thyroid cancer; NMTC: non-medullary thyroid cancer; PTC: papillary thyroid carcinoma; TC: thyroid cancer.

\section{Author contributions}

Artur Kowalik (AK) conceived the project, designed the experiments, interpreted genetic and clinical data, and wrote the manuscript. Danuta Gąsior-Perczak recruited patients, collected clinical data, and wrote the manuscript. AK designed the genetic analysis, with assistance from Malgorzata Chlopek and Martyna Gromek (MG). MG conducted genetic studies, collected and prepared genetic data for analysis and wrote the manuscript. Monika Siołek, Agnieszka Walczyk, and Iwona Pałyga were responsible for patient recruitment and clinical data collection. Janusz Kopczyński was responsible for clinical data collection. Ryszard Mężyk performed statistical analyses. Aldona Kowalska conceived the project and wrote the manuscript. Stanisław Góźdź conceived the project and was responsible for final approval of the manuscript. All authors read and approved the final manuscript.

\section{ACKNOWLEDGMENTS}

We thank all patient and volunteer blood donors for their contributions.

\section{CONFLICTS OF INTEREST}

The authors declare no conflicts of interest.

\section{FUNDING}

The study was financially supported by the Holycross Cancer Center.

\section{REFERENCES}

1. Siegel RL, Miller KD, Jemal A. Cancer statistics, 2016. CA Cancer J Clin. 2016; 66:7-30.

2. Kowalska A, Walczyk A, Kowalik A, Pałyga I, Trybek T, Kopczyński J, Kajor M, Chrapek M, Pięciak L, Chłopek M, Góźdź S, Kamiński G. Increase in papillary thyroid cancer incidence is accompanied by changes in the frequency of the BRAF V600E mutation: a singleinstitution study. Thyroid. 2016; 26:543-551.

3. Vriens MR, Suh I, Moses W, Kebebew E. Clinical features and genetic predisposition to hereditary nonmedullary thyroid cancer. Thyroid. 2009; 19:1343-1349.

4. Xing M. Molecular pathogenesis and mechanisms of thyroid cancer. Nat Rev Cancer. 2013; 13:184-199.
5. Gara SK, Jia L, Merino MJ, Agarwal SK, Zhang L, Cam M, Patel D, Kebebew E. Germline HABP2 mutation causing familial nonmedullary thyroid cancer. N Engl J Med. 2015; 373:448-455.

6. Zhang T, Xing M. HABP2 G534E mutation in familial nonmedullary thyroid cancer. J. Natl Cancer Inst. 2016; 108:djv415.

7. Sponziello M, Durante C, Filetti S. HABP2 mutation and nonmedullary thyroid cancer. N Engl J Med. 2015; 373:2085-2086.

8. Tomsic J, He H, de la Chapelle A. HABP2 mutation and nonmedullary thyroid cancer. N Engl J Med. 2015; 373:2086.

9. Zhao X, Li X, Zhang X. HABP2 mutation and nonmedullary thyroid cancer. N Engl J Med. 2015; 373:2084.

10. Zhou EY, Lin Z, Yang Y. HABP2 mutation and nonmedullary thyroid cancer. N Engl J Med. 2015; 373:2084-2085.

11. Tomsic J, Fultz R, Liyanarachchi S, He H, Senter L, de la Chapelle A. HABP2 G534E variant in papillary thyroid carcinoma. PLoS One. 2016; 11:e0146315.

12. Sahasrabudhe R, Stultz J, Williamson J, Lott P, Estrada A, Bohorquez M, Palles C, Polanco-Echeverry G, Jaeger E, Martin L, Magdalena Echeverry M, Tomlinson I, CarvajalCarmona LG, TCUKIN. The HABP2 G534E variant is an unlikely cause of familial non-medullary thyroid cancer. J Clin Endocrinol Metab. 2015:jc20153928.

13. Ruiz-Ferrer M, Fernández RM, Navarro E, Antiñolo G, Borrego S. G534E variant in HABP2 and nonmedullary thyroid cancer. Thyroid. 2016; 26:987-988.

14. Weeks AL, Wilson SG, Ward L, Goldblatt J, Hui J, Walsh JP. HABP2 germline variants are uncommon in familial nonmedullary thyroid cancer. BMC Med Genet. 2016; 17:60.

15. Alzahrani AS, Murugan AK, Qasem E, Al-Hindi H. HABP2 gene mutations do not cause familial or sporadic nonmedullary thyroid cancer in a highly inbred middle Eastern population. Thyroid. 2016; 26:667-671.

16. Charkes ND. On the prevalence of familial nonmedullary thyroid cancer in multiply affected kindreds. Thyroid. 2006; 16:181-186.

17. Wang X, Cheng W, Li J, Su A, Wei T, Liu F, Zhu J. Endocrine tumours: familial nonmedullary thyroid carcinoma is a more aggressive disease: a systematic review and meta-analysis. Eur. J. Endocrinol. 2015; 172:R253-262.

18. Nagy R, Ringel MD. Genetic predisposition for nonmedullary thyroid cancer. Horm Cancer. 2015; 6:13-20.

19. Bohórquez ME, Estrada AP, Stultz J, Sahasrabudhe R, Williamson J, Lott P, Duque CS, Donado J, Mateus G, Bolaños F, Vélez A, Echeverry M, Carvajal-Carmona LG. The HABP2 G534E polymorphism does not increase nonmedullary thyroid cancer risk in Hispanics. Endocr Connect. 2016; 5:123-127. 